\title{
Krüppel-like Factor 5 contributes to pulmonary artery smooth muscle proliferation and resistance to apoptosis in human pulmonary arterial hypertension
}

Audrey Courboulin ${ }^{\dagger}$, Véronique L Tremblay ${ }^{\dagger}$, Marjorie Barrier, Jolyane Meloche, Maria Helena Jacob, Mathilde Chapolard, Malik Bisserier, Roxane Paulin, Caroline Lambert, Steeve Provencher and Sébastien Bonnet ${ }^{*}$

Background: Pulmonary arterial hypertension (PAH) is a vascular remodeling disease characterized by enhanced proliferation of pulmonary artery smooth muscle cell (PASMC) and suppressed apoptosis. This phenotype has been associated with the upregulation of the oncoprotein survivin promoting mitochondrial membrane potential hyperpolarization (decreasing apoptosis) and the upregulation of growth factor and cytokines like PDGF, IL-6 and vasoactive agent like endothelin-1 (ET-1) promoting PASMC proliferation. Krüppel-like factor 5 (KLF5), is a zincfinger-type transcription factor implicated in the regulation of cell differentiation, proliferation, migration and apoptosis. Recent studies have demonstrated the implication of KLF5 in tissue remodeling in cardiovascular diseases, such as atherosclerosis, restenosis, and cardiac hypertrophy. Nonetheless, the implication of KLF5 in pulmonary arterial hypertension (PAH) remains unknown. We hypothesized that KLF5 up-regulation in PAH triggers PASMC proliferation and resistance to apoptosis.

Methods and results: We showed that KFL5 is upregulated in both human lung biopsies and cultured human PASMC isolated from distal pulmonary arteries from PAH patients compared to controls. Using stimulation experiments, we demonstrated that PDGF, ET-1 and IL-6 trigger KLF-5 activation in control PASMC to a level similar to the one seen in PAH-PASMC. Inhibition of the STAT3 pathway abrogates KLF5 activation in PAH-PASMC. Once activated, KLF5 promotes cyclin B1 upregulation and promotes PASMC proliferation and triggers survivin expression hyperpolarizing mitochondria membrane potential decreasing PASMC ability to undergo apoptosis.

Conclusion: We demonstrated for the first time that KLF5 is activated in human PAH and implicated in the proproliferative and anti-apoptotic phenotype that characterize PAH-PASMC. We believe that our findings will open new avenues of investigation on the role of KLF5 in PAH and might lead to the identification of new therapeutic targets.

Keywords: Pulmonary arterial hypertension, KLF5, STAT3, proliferation, apoptosis.

\section{Background}

Pulmonary arterial hypertension (PAH) is a vascular disease that is mainly restricted to small pulmonary arteries. PAH occurs in rare idiopathic and familial forms, but is more commonly part of syndromes

\footnotetext{
* Correspondence: sebastien.bonnet@crhdq.ulaval.ca

† Contributed equally

Department of Medicine, Faculty of Medicine, Laval University, Quebec QC,
} Canada

C Biomed Central

(c) 2011 Courboulin et al; licensee BioMed Central Ltd. This is an Open Access article distributed under the terms of the Creative Commons Attribution License (http://creativecommons.org/licenses/by/2.0), which permits unrestricted use, distribution, and reproduction in any medium, provided the original work is properly cited. associated with connective tissue diseases, anorexigen use, HIV or congenital heart disease. This syndrome of obstructed, constricted small pulmonary arteries (PA) has been attributed to abnormalities in the blood content of some neurotransmitters and cytokines, namely increases in serotonin, IL-6, PDGF and endothelin-1 [1-4]. We recently demonstrated that the increase in these circulating vasoactive agents triggers in pulmonary artery smooth muscle cells (PASMC) the activation of 
the nuclear factor of activated T-cells (NFAT) contributing to increase $\left[\mathrm{Ca}^{2+}\right]_{\mathrm{i}}$-mediated PASMC proliferation $[5,6]$. Moreover, we showed a sustained increase in the oncoprotein survivin, decreasing mitochondrial-dependent apoptosis [7]. The fact that the PAH phenotype is preserved in cultured PASMC isolated from $\mathrm{PAH}$ patients suggests that the PAH phenotype is sustained independently of the circulating growth factors or agonists but requires genetic remodeling processes $[8,9]$. Moreover, despite recent therapeutic advances such as endothelin-1 receptor blockers (e.g. bosentan) [10], type 5 phosphodiesterase inhibitors (e.g. sildenafil) [11] or PDGF receptor blockers (e.g. imanitib) [12], mortality rates remain high [13].

Krüppel-like factor 5 is a zinc finger transcription factor that belongs to a family known as the Sp/KLF factors, and is implicated in important biological functions including cell proliferation, apoptosis, development, and oncogenic processes [14-16]. In Vascular Smooth Muscle Cells (VSMC) KLF5 regulates expression of the embryonic form of smooth muscle myosin heavy chain (SMemb/NMHC-B), which is selectively expressed in the proliferative dedifferentiated smooth muscle phenotype. In systemic vessels, KLF5 is expressed in proliferating smooth muscle cells of coronary artery lesions [17], and expression of this factor in lesions is clinically associated with restenosis and cardiac allograft vasculopathy [17]. KLF5 expression is therefore associated with proliferating smooth muscle cells in the cardiovasculature $[18,19]$. However, it had yet to be shown whether KLF5 is activated in PAH-PASMC and whether it's implicated in PASMC proliferation and apoptosis.

\section{Materials and methods}

All experiments were performed in accordance to the Université Laval's Ethic and Biosafety Committee (protocol number 20142) and the Centre Hospitalier Universitaire de Québec's Ethic's Committee.

\section{Human tissue samples}

All patients gave written informed consent before the study. Healthy lung tissues (controls) were obtained during lung resection for tumors. Only the healthy parts of the lungs were used in this study. All the PAH tissues were from lung explants from transplant or autopsy (Table 1).

\section{Cell culture}

Cells were used in the first to sixth passage. Pulmonary arterial endothelial cell (PAEC), isolated form healthy human pulmonary arteries, were bought form the Cell application inc. (\#302K-05a). PAH-PASMC were obtained from $\approx 1,5 \mu \mathrm{m}$-diameter small pulmonary arteries from 2 males with iPAH ( 31 and 48 years old) and 1 female with PAH group 1 ( 54 years old) from lung explants. Age and sex matched control PASMC (3 males 45; 21; 64 years old and 2 females 17 and 35 years old)) were used. All patients had right catheterization that confirmed pulmonary hypertension (mean PAP greater than $25 \mathrm{mmHg}$ at rest). PASMC were grown in high-glucose DMEM supplemented with 10\% Fetal Bovine Serum (Gibco, Invitrogen,

Table 1 Patients providing tissue

\begin{tabular}{|c|c|c|c|c|c|c|c|}
\hline & Patient type & Sex & Age & Mean PA pressure $(\mathrm{mmHg})$ & Medications & PVR (dyne*sec) $/ \mathrm{cm}^{5}$ & Lung tissue \\
\hline 1 & Control (Benign tumor) & $\mathrm{F}$ & 35 & ND & None & ND & Yes \\
\hline 2 & Control (Lung Cancer) & $\mathrm{F}$ & 38 & ND & None & ND & Yes \\
\hline 3 & Control (Benign tumor) & M & 45 & ND & None & ND & Yes \\
\hline 4 & Control (Lung Cancer) & M & 51 & ND & None & ND & Yes \\
\hline 5 & Control (Hodgkin) & $M$ & 48 & ND & None & ND & Yes \\
\hline 6 & Control (Benign tumor) & $\mathrm{F}$ & 44 & ND & None & ND & Yes \\
\hline 7 & Control (Lung Cancer) & $\mathrm{F}$ & 47 & ND & None & ND & Yes \\
\hline 8 & Control (Lung Cancer) & $\mathrm{F}$ & 50 & ND & None & ND & Yes \\
\hline 9 & IPAH & $\mathrm{F}$ & 58 & 56 & Epoprostenol/Lasix/Coumadin & 1709 & Yes \\
\hline 10 & iPAH & $\mathrm{F}$ & 36 & 67 & Epoprostenol/Lasix/Coumadin & 2274 & Yes \\
\hline 11 & SSC-PAH & $\mathrm{F}$ & 55 & 48 & Epoprostenol/Lasix/Coumadin & 980 & Yes \\
\hline 12 & PAH group1 & $\mathrm{F}$ & 64 & 59 & Bosentan/Lasix & 926 & Yes \\
\hline 13 & PAH group1 & M & 72 & 39 & Lasix/Sitaxsentan & 550 & Yes \\
\hline 14 & PAH group1 & $M$ & 58 & 42 & Epoprostenol/Lasix/Coumadin & 991 & Yes \\
\hline 15 & PAH group1 & $\mathrm{F}$ & 51 & 51 & Lasix/coumadin & 1199 & Yes \\
\hline 16 & PAH group1 & $\mathrm{F}$ & 48 & 73 & Epoprostenol/Lasix & 1800 & Yes \\
\hline 17 & PAH group1 & $\mathrm{F}$ & 51 & 41 & Lasix & 990 & Yes \\
\hline 18 & PAH group1 & $\mathrm{F}$ & 68 & 37 & Lasix/coumadin/Norvasc & 544 & Yes \\
\hline
\end{tabular}


Burlington, ON, Canada) and 1\% antibiotic/antimytotic (Gibco, Invitrogen, Burlington, ON, Canada) [20]. STAT3 was inhibited by a specific siRNA $20 \mathrm{nM}$ for $48 \mathrm{~h}$ (Applied Biosystems Canada) and KLF5 was inhibited by a specific siRNA (30 nM for $48 \mathrm{~h}$; Applied Biosystems Canada). Control PASMC were exposed to either PDGF (30 nG/ $\mathrm{mL})$; endothelin-1 (10 nM) or IL6 (20 ng/mL) (all from EMB Canada). All experiments were realized with a proper control siRNA scramble (siScr).

\section{In vivo model rats}

Male Sprague-Dawley rats were used in this study. Monocrotaline (Sigma) was injected s.c. $(60 \mathrm{mg} / \mathrm{kg})$ to induce PAH. Once PAH is established (2 weeks post MCT injection), rats were treated by nebulization directly in the lungs of KLF5 siRNA $(20 \mu \mathrm{M})$. PA pressure measurements have been realized by right heart catheterization to evaluate $\mathrm{PAH}$ severity.

\section{Quantitative RT-PCR}

To measure KLF5 expression (using assay from Applied Biosystem), total mRNA was extracted from PAHPASMC or control PASMC using trizol protocol. Quantification of mRNA expression was performed as previously described [6].

\section{Western Blot}

Total protein extraction was realized on PASMC. KLF5 (Millipore; 1/500), PY705-STAT3 (Cell signaling; 1/ 1000), STAT3 (Cell signaling; 1/1000), Survivin (Cell signaling; 1/1000) and Cyclin B1 (Sigma Aldrich; 1/ 1000) were quantified and normalized to the smooth muscle actin (Santa cruz; 1/400) as previously described [6]. Evaluation for PY705-STAT3/STAT3 and KLF5/ SM-actin were obtained from the same gel after stripping (30 min at 50 degrees).

\section{Confocal microscopy}

TMRM, TUNEL, PCNA, Ki67 and AnnexinV were measured as previously described $[6,21]$.

\section{Statistical Analysis}

Values are expressed as fold change or mean \pm SEM. Unpaired Student's $t$ tests were used for comparisons between two means. For comparisons between more than two means we used one-way ANOVA followed by a Dunn's test. A $P$ value $<0.05$ was considered statistically significant (and indicated with asterisks *).

\section{Results}

KLF5 is upregulated in human and rodent pulmonary hypertension

To investigate the expression pattern of KLF5 in normal and pulmonary hypertensive lungs, we examined KLF5 expression levels in 1) lung biopsies from 10 individuals with non-familial PAH compared to biopsies from 8 individuals without pulmonary hypertension, 2) lungs from 5 rats with monocrotaline-induced pulmonary hypertension compared to 10 control littermates (Figure 1A). We found increased levels of KLF5 in human and rodent pulmonary hypertensive lung tissues compared to normotensive lung samples. Within the lungs, KLF5 upregulation in PAH is confined to the distal PA $(<400 \mu \mathrm{m})$ (Figure 1A).

To test whether KLF5 upregulation is correlated with disease progression, we studied humans and rats with varying degrees of PAH. In both human subjects and rodents, KLF5 levels in the lung correlated directly with the severity of PAH, as measured by pulmonary vascular resistance (PVR) in humans and mean PA pressure in rodents (Figure 1B). Our results indicate that levels of KLF5 correlate with the severity of PAH in humans and experimental pulmonary hypertension.

To determine whether KLF5 expression in the pulmonary circulation is restricted to PASMC we measured KLF5 mRNA in human PASMC and PAEC. As shown in Figure 1C, KLF5 is preferentially expressed in PASMC. This finding was confirmed by co-localization studies between KLF5 and smooth muscle actin (marker of smooth muscle cell) or VE cadherin (marker of endothelial cells) in human lung biopsies. As shown, a greater co-localization was found between KLF5 and smooth muscle actin than KLF5 and VE cadherin. This finding confirms our qRT-PCR data and thus, that KLF5 is significantly more expressed in PASMC than PAEC (Figure 1C). Hence, for the rest of the study we have focused our research on PASMC. To determine whether KLF5 is upregulated in PAH-PASMC we used human cultured PASMC in the first to third passage. PAHPASMC were obtained from $\approx 1,500 \mu \mathrm{m}$-diameter small pulmonary arteries from 2 males with iPAH (31 and 48 years old) and 1 female with PAH group 1 (lupus; 54 years old) from lung explants [7]. All patients had right catheterization that confirmed pulmonary hypertension (mean PAP greater than $25 \mathrm{mmHg}$ at rest). Age and sex matched control PASMC (3 males $45 ; 21 ; 64$ years old and 2 females 17 and 35 years old)) were used. KLF5 upregulation between control and $\mathrm{PAH}$ was confirmed by qRT-PCR and Western blot (WB) (Figure 1C). Note that no significant differences in KLF5 expression was found among the control patients and among the PAH patients (not shown). Therefore for the rest of the study all 5 control-PASMC and the 3 PAH-PASMC cell lines were used for every cell-based experiment.

\section{KLF5 is activated in PAH-PASMC through a STAT3 dependent mechanism}

The Signal Transducers and Activators of Transcription (STATs) family is composed of 7 isoforms (STAT1; 2; 3; 


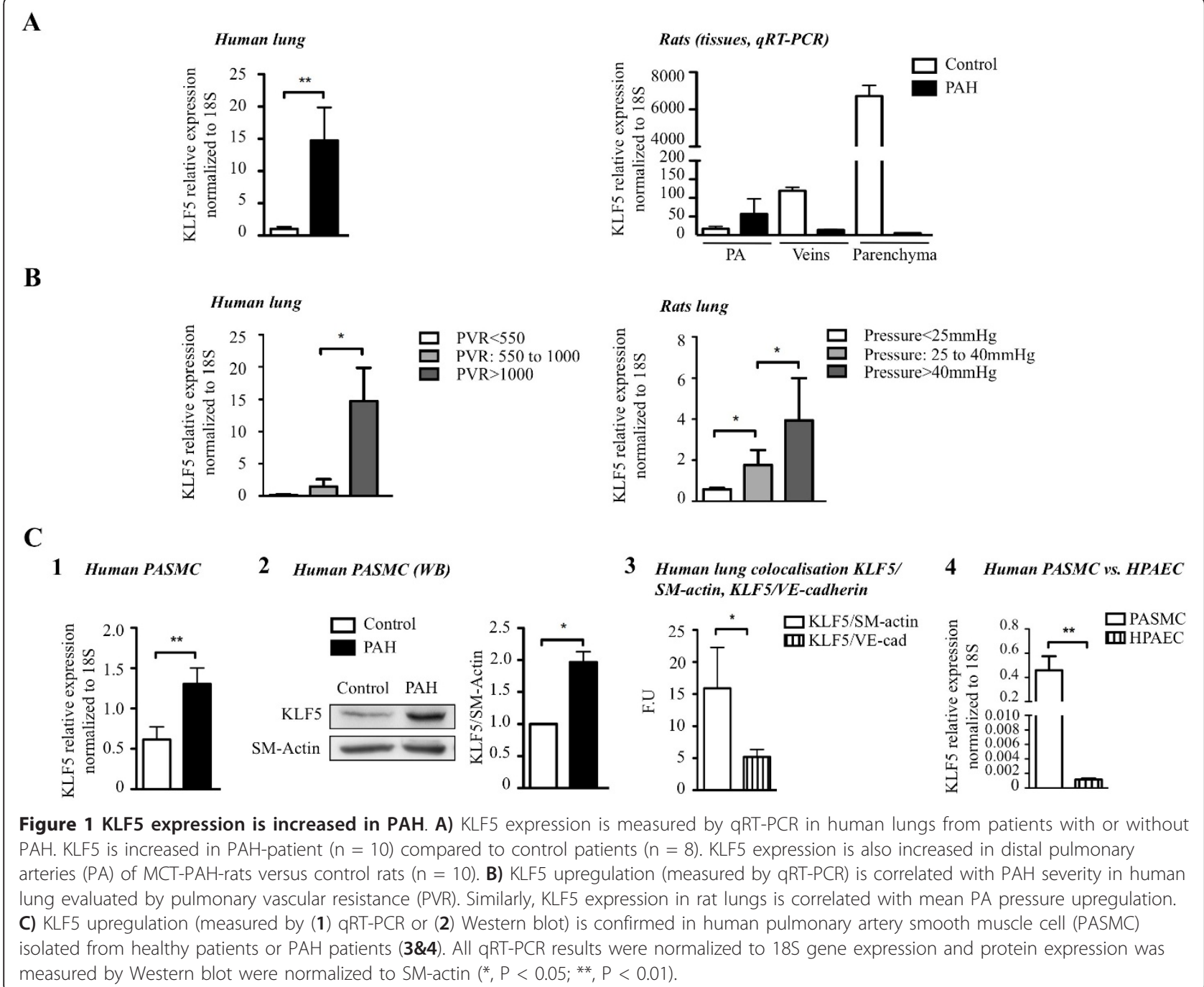

4; 5A; 5B and 6) with STAT3 being the most important one in cardiovascular diseases $[22,23]$. STATs are activated (i.e. phosphorylated) by either the Janus-activated kinase (JAKs) or the Src family kinases in response to cytokines (like IL-6, TNF...) [24], growth factors (like PDGF...) [24] or agonists (like ET1, AngII...) [25], all of which are implicated in PAH [26-28]. Interestingly, a recent study in stem cells revealed a putative regulation of KLF5 by STAT3 [29]. To demonstrate the implication of STAT3 in the regulation of KLF5 expression in PAH, KLF5 expression was measured in PAH-PASMC and control PASMC in the presence or absence of STAT3 siRNA. Compared to siScr treated PAH-PASMC, siSTAT3 treated PAH-PASMC had significantly reduced KLF5 expression (Figure 2A). These findings suggest that STAT3 activation accounts for KLF5 expression in PAH-PASMC. To further confirm the implication of STAT3/KLF5 axis in PAH, healthy-PASMC were treated for $48 \mathrm{~h}$ with increasing doses of pro-PAH factors [26-28] like ET1 (10 nM), PDGF (30 ng.mL $\left.{ }^{-1}\right)$ and IL-6 $\left(20\right.$ ng. $\left.\mathrm{mL}^{-1}\right)$. As expected, pro-PAH factors, increased STAT3 activation (PY705-STAT3/STAT3 ratio) in ET1, PDGF and IL-6 treated PASMC respectively (Figure 2B). Interestingly, we found that in healthy-PASMC treated with pro-PAH factors (ET1, PDGF or IL-6) the activation of STAT3 promotes KLF5 expression (Figure 2B).

\section{KLF5 inhibition decreases PAH-PASMC proliferation and resistance to apoptosis}

To study the effect of KLF5 on PASMC proliferation and apoptosis in vitro, cultured human PAH-PASMC were either exposed to 10\% FBS to promote proliferation or $0.1 \%$ FBS to promote apoptosis [6]. When compared to control PASMC containing a low level of KLF5, PAH-PASMC displayed higher cell proliferation rate and resistance to induced apoptosis (Figure $3 \& 4$ ). 


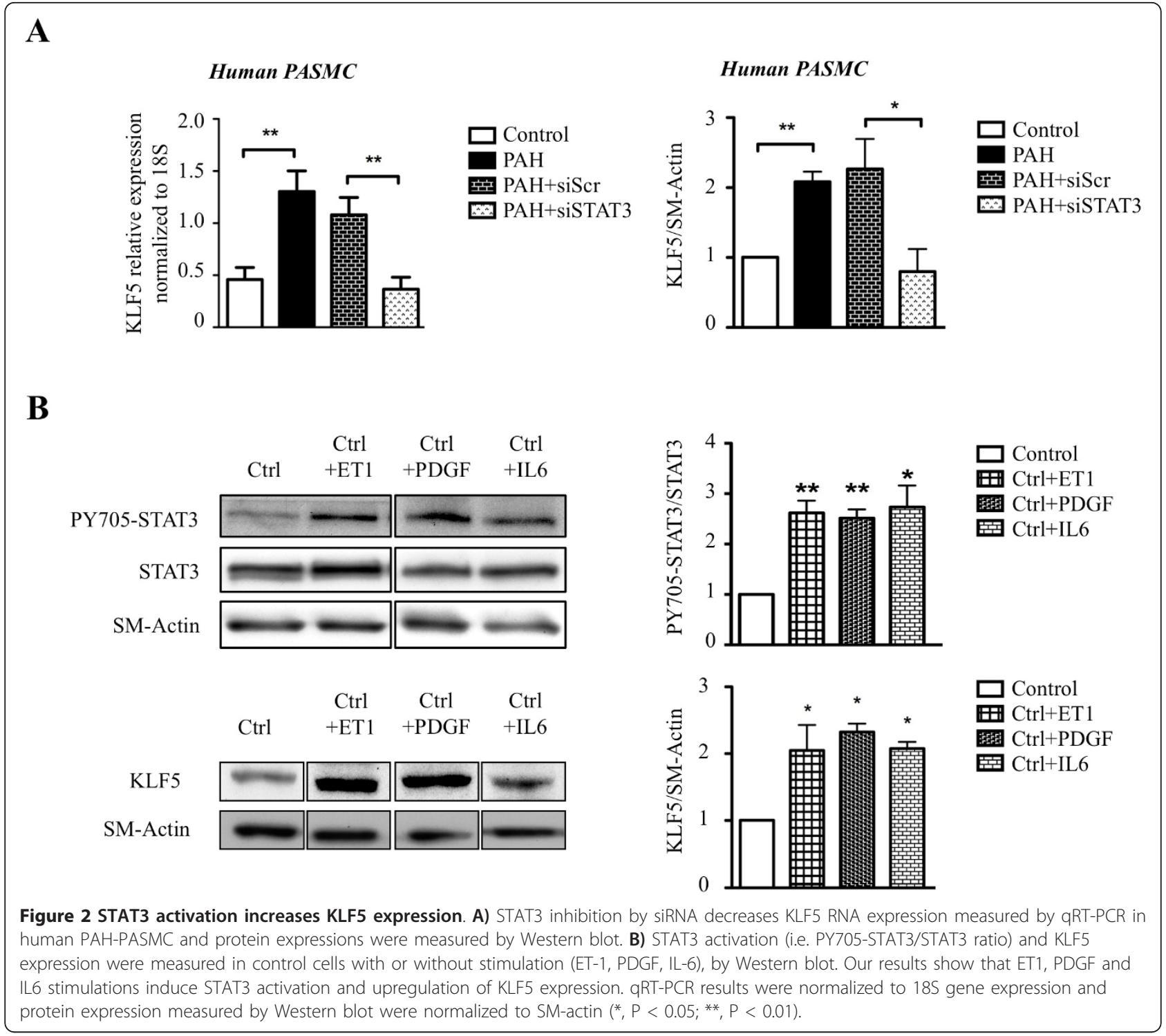

Using Ki67 and PCNA, we measured the effect of KLF5 inhibition on PAH-PASMC proliferation. The KLF5 inhibition using siRNA in PAH-PASMC decreases proliferation to the level seen in control-PASMC (Figure 3A\&3B). Note that siScr has no effect. The increased proliferation seen in PAH-PASMC is associated with a KLF-5-dependent up-regulation of cyclin B1, confirming previous findings in systemic vessels [30] and cancer [31] (Figure 3C). Moreover, we confirmed that siKLF5 decreased significantly KLF5 (Figure 3D).

Resistance to apoptosis observed in PAH-PASMC has been linked to mitochondrial membrane potential $\left(\Delta \Psi_{\mathrm{m}}\right)$ hyperpolarization, which would block the release of pro-apoptotic mediators like cytochrome $c[6,20,21]$. Using tetramethylrhodamine methyl ester (TMRM), we measured whether KLF5 inhibition can affect mitochondrial hyperpolarization. We observed that KLF5 inhibition in PAH-PASMC depolarizes $\Delta \Psi_{\mathrm{m}}$ to a level similar to that observed in control-PASMC (Figure 4A). Finally, mitochondrial depolarization induced by KLF5 inhibition in PAH-PASMC increases serum starvation-induced apoptosis (TUNEL, annexin V) (Figure $4 \mathrm{~B} \& 4 \mathrm{C})$. We previously published that $\Delta \Psi_{\mathrm{m}}$ hyperpolarization was associated to the upregulation of the oncoprotein survivin and that survivin inhibition in $\mathrm{PAH}-$ PASMC reverses mitochondrial hyperpolarization, promoting apoptosis. Nonetheless, the mechanism accounting for survivin expression in PAH-PASMC remains elusive. Recently, in cancer, KLF5 has been shown to promote survivin expression. To determine whether the survivin upregulation in PAH-PASMC is KLF5 dependent, survivin expression was measured in PAH-PASMC 


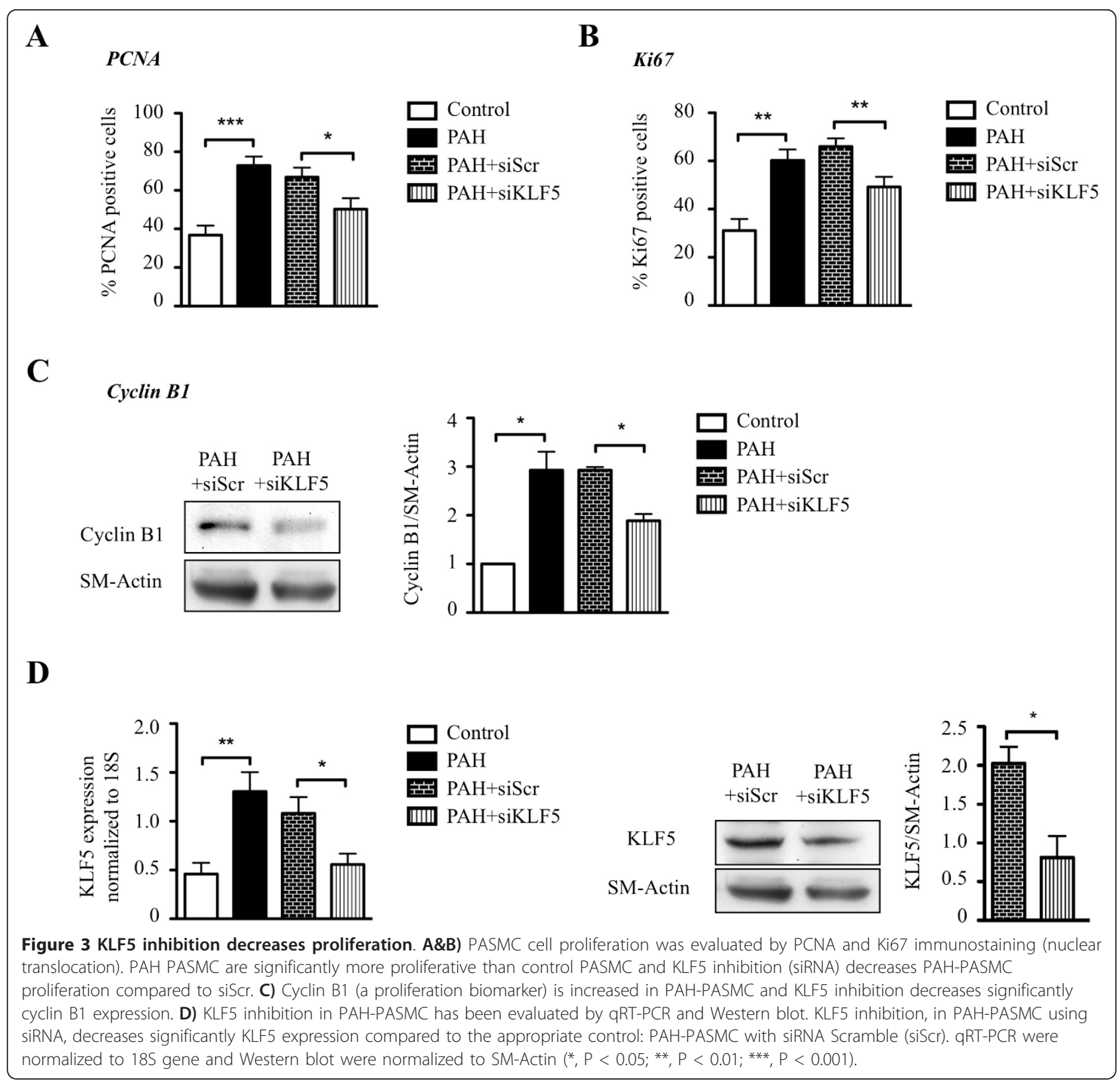

in presence and absence of KLF5 siRNA. We found that KLF5 inhibition decreases survivin expression (Figure 4D) and thus provides a putative mechanism accounting for the KLF5-dependent $\Delta \Psi_{\mathrm{m}}$ hyperpolarization seen in PAH-PASMC.

\section{KLF5 inhibition in vivo improves PAH}

To confirm the STAT3-dependent regulation of KLF5, in vivo rats were injected with monocrotaline for 4 weeks. Once PAH established (increased PA pressure and increased right ventricle wall hypertrophy (RVH)), rats were nebulized with either siSTAT3 or siScr for 2 weeks. Effects on PAH (mean PA pressure; RVH) were measured by right catheterization and Fulton index. KLF5 expression was measured immunofluorescence in distal PA (Figure 5B). As shown, siSTAT3 decreases KLF5 expression and PAH in distal PA, confirming our in vitro findings (Figure 2A). Note that the efficiency of our therapeutic intervention was confirmed i.e. siSTAT3 significantly decreases STAT3 in distal PA. Among all the rats treated with siSTAT3, the rats showing the greater KLF5 downregulation had the lower mean PA pressure, suggesting that KLF5 is implicated in the etiology of PAH. To determine whether KLF5 has direct therapeutic potential for $\mathrm{PAH}$, monocrotaline rats (with established PAH 2 weeks post injection) were nebulized 


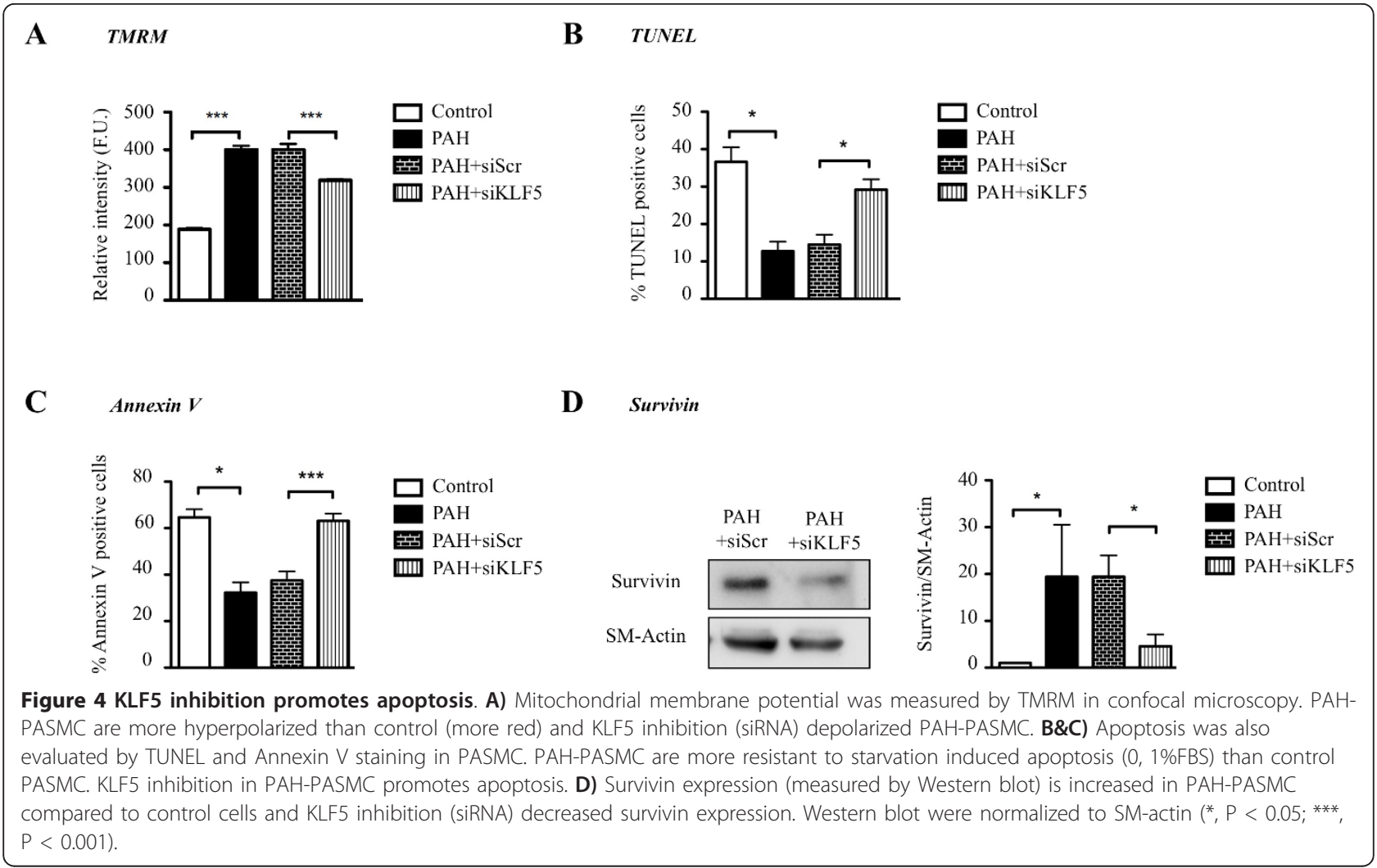

with either siKLF5 or siScr for 2 week. Efficiency of our intervention was confirmed in distal PA where KLF5 expression is significantly decreased (Figure 5B). As shown, KLF5 inhibition improves PAH by reducing $\mathrm{RVH}$ and mean PA pressure (Figure 5A).

\section{Discussion}

Here we show that KLF5 is etiologically associated with the development of PAH, and believe that we have opened a new avenue for PAH treatment. KLF5 is expressed in established human and experimental PAH. Its expression is dependent of the activation of STAT3. We provided evidences that KLF5 inhibition improves PAH by 1) the inhibition of cyclin B1 and PASMC proliferation and 2) the depolarization of PASMC mitochondria by inhibiting survivin activation thus increasing apoptosis. These findings not only confirm what has been shown in cancer $[16,31,32]$ and systemic vascular smooth muscle cells $[33,34]$ in which KLF5 inhibition decreases proliferation through cell cycle protein inhibition like cyclin B1 and p21 upregulation, but also provide a better demonstration of the involvement of KLF5 in mitochondrial-dependent apoptosis. In fact, we provide for the first time evidences that KLF5 is implicated in mitochondrial membrane potential regulation through the upregulation of the oncoprotein survivin. We have previously extensively demonstrated the mechanism of mitochondrial membrane potential regulation by survivin in PAH-PASMC [7], but had not elucidated the mechanism accounting for its upregulation. Our new findings propose that KLF5 activation might explain such upregulation. In fact, we showed that KLF5 inhibition decreases survivin expression in $\mathrm{PAH}-$ PASMC. This is associated with a significant mitochondrial depolarization. This finding could be of great therapeutic interest as it provides a new insight on the regulation of survivin that is implicated in many cancer and cardiovascular diseases [35-39]. Thus, our findings might not be limited to PAH but can be extend to many other diseases including cancer. To this end, a recent report has demonstrated a link between KLF5 and survivin in cancer [40].

Previous studies reported that KLF5 is implicated in embryonic stem cells and VSMC differentiation contributing to vascular lesions [34,41]. This aspect might be implicated in the vascular lesions seen in PAH patients. Indeed, vascular lesions such as remodeled arteries and plexiform lesions are seen in patients with PAH. Studies have demonstrated the implications of abnormal stem cells in this phenomenon [42]. KLF5 implication in such processes cannot be ruled out.

The activation of KLF5 axis that we described likely has a multifactorial etiology in PAH. However, KLF5 might be a critical integrator of multiple signaling 
$\mathbf{A}$

Mean PA pressure

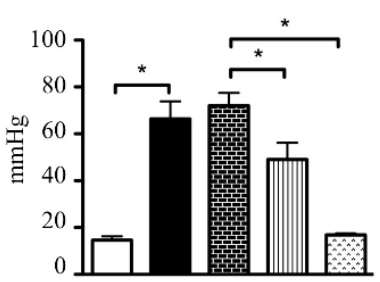

RV hypertrophy (Fulton index)

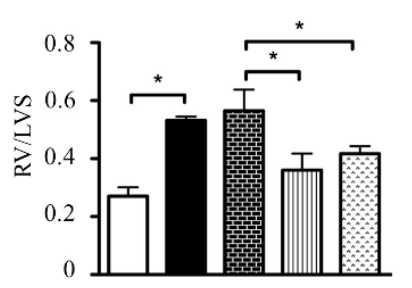

Pulmonary vascular resistance

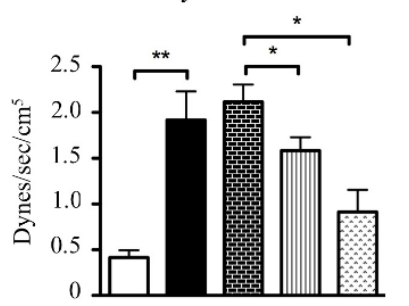

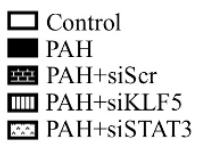

PAH+siSTAT3

B
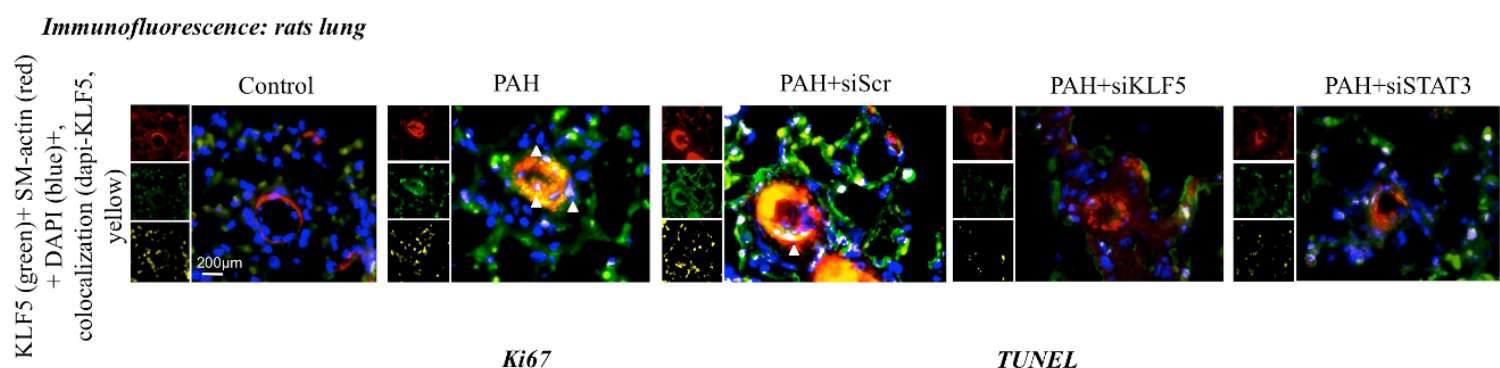

Ki67

TUNEL
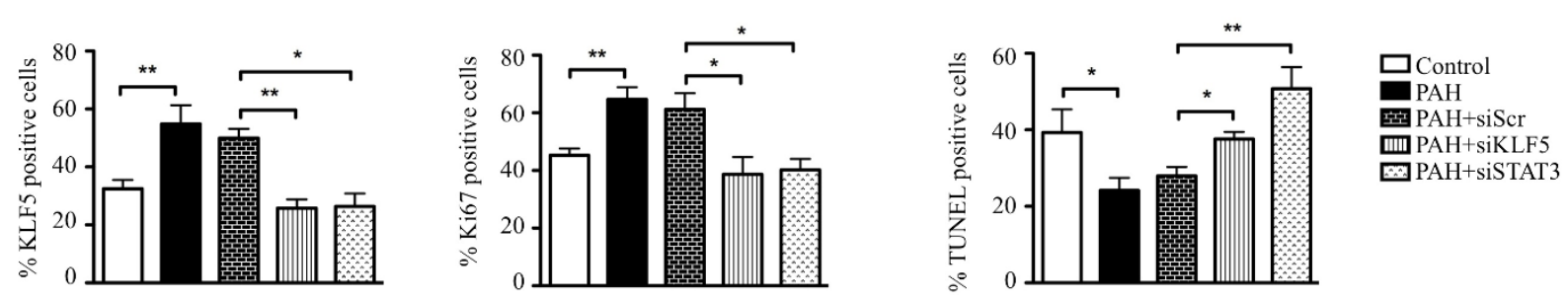

Figure 5 In vivo, KLF5 inhibition reverses PAH development. A) Mean PA pressure was measured on closed chest rats by right catheterization. The mean PA pressure is increased significantly in PAH, whereas KLF5 and STAT3 inhibition (siRNA) decrease the pulmonary pressure. The right ventricular hypertrophy, evaluated by Fulton index measurement, is reversed with KLF5 and STAT3 inhibition. Pulmonary Vascular Resistances (PVR) are increased in PAH rats and KLF5 or STAT3 inhibition decreased them. B) KLF5 activation has been evaluated in distal pulmonary arteries from rats by immunofluorescence. Nuclear colocalization of KLF5 is increased in PAH-PASMC from distal PA compared to healthy PA and KLF5 and STAT3 inhibition decrease KLF5 translocation. Proliferation and apoptosis have been evaluated by

immunofluorescence (respectively by Ki67 and TUNEL). Proliferation increases in PAH-PA compared to control PA was decreased after treatment with siKLF5 or siSTAT3. Apoptosis resistance of the PA-PAH is reversed after KLF5 and STAT3 inhibition $\left(^{*}, \mathrm{P}<0.05 ;{ }^{* *}, \mathrm{P}<0.01 ;{ }^{* *}, \mathrm{P}<0.001\right)$.

pathways and its downstream effects might explain several and important features of PAH. In vivo, endothelial dysfunction is recognized as one of the earliest abnormalities in $\mathrm{PAH}$, resulting in a well-recognized imbalance of endothelium-derived vasoactive factors; with increased vasoconstrictors (like endothelin [28], thromboxane [1] and decreased vasodilators (like NO or prostacyclin [1]). Recently, KLF5 has been shown to be implicated in endothelial dysfunction [43]. In addition, increased circulating growth factor (like PDGF) [27] and cytokines (like IL-6, MCP-1...) [26] have been reported in $\mathrm{PAH}$, and there is numerous evidences demonstrating the implication of KLF5 in both growth factor and cytokines production regulation [18,43-45].

Finally, we have previously extensively demonstrated the implication of HIF-1 in triggering mitochondrial and $\left[\mathrm{Ca}^{2+}\right]_{\mathrm{i}}$ dysfunction sustaining the pro-proliferative and anti-apoptotic phenotype seen in PAH-PASMC $[5,46]$. Interestingly, Mori et al. have shown that cooperation between HIF-1 and KLF5 might exist [47]. Indeed, KLF5 inhibition decreases the expression of several HIF-1regulated genes in cancer cells, while HIF-1 inhibition affects KLF5 expression [47]. These findings in cancer cells implied that interaction between HIF-1/KLF5 in PAH-PASMC might exist. Thus, all these reports suggest that KLF5 might play a critical role in the etiology of PAH. Surprisingly no studies have been performed on the topic.

The mechanisms accounting for KLF5 upregulation in PAH remain to be established. Recently, a study from Cheng et al [48] has suggested the implication of the microRNA 145 (miR-145). In their study, they provided evidences that the downregulation of miR-145 promotes vascular neointimal lesion formation through the 
upregulation of KLF5. Nonetheless, downregulation of miR-145 in PAH, has not been demonstrated [49]. We believe that one of the initial events in PAH, regardless of the specific cause, is the activation of the miR-204/STAT3 axis [50], which increases KLF5 expression (Figure 2), supporting the notion that KLF5 induction is an early event during PAH. Thus, KLF5 upregulation can be caused by many diverse conditions that lead to $\mathrm{PAH}$ in addition to hypoxia, including growth factors, vasoactive molecules [50,51], or viral infection of PASMC [52]. The fact that STAT3 activation may occur only in pulmonary, not systemic, vasculature [50] explains the selective induction of KLF5 in the pulmonary circulation. This finding strengthens the argument that KLF5 therapeutic targeting may achieve relative selectivity for the pulmonary circulation in $\mathrm{PAH}$. Although many more experiments are needed in order to determine whether this regulation is direct or indirect, this finding is of great interest. In fact, STAT3 has been associated with many feature of PAH including BMPR2 downregulation (hallmark of PAH) [53]. Involvement of KLF5 in the STAT3-mediated effects in PAH will certainly open new avenues of investigation. Finally, we showed that both indirect (through STAT3 inhibition) and direct KLF5 inhibition (nebulized siRNA) improve PAH. Nonetheless, we should note that STAT3 inhibition showed a greater efficiency than siKLF5. This is not surprising as STAT3 has been implicated in many features characterizing PAH, including miRNA such as miR-204, Pim1; NFAT; BMPR2 [6,50,51,53]

\section{Conclusion}

Although our findings will need to be repeated in greater amount of patients and at different stages of PAH to have a better understanding of the role of KLF5 in $\mathrm{PAH}$, nonetheless our study suggests for the first time the implication of KLF5 in the etiology of human $\mathrm{PAH}$. Moreover we provide evidences that its activation might account for many feature seen in $\mathrm{PAH}$, including PASMC proliferation, mitochondrial hyperpolarization, survivin expression and resistance to apoptosis. Because, pulmonary arterial hypertension is a rapidly lethal disease for which treatments are limited, we believe that our findings will open the door to new avenues of investigation and potentially future therapies for PAH.

\footnotetext{
Acknowledgements

This work has been funded by the Canadian Institutes of Health Research (CIHR) to S.B. S.B hold Canada Research Chairs and Dr Jacob was supported by CAPES (Brazilian Research Agency). AC, JM and RP are recipients of Graduate Scholarships from La Société Qébécoise d'Hypertension Artérielle (SQHA).
}

\section{Authors' contributions}

AC contributed in all the data experiments, analysis and elaborated the figures. VLT performed the Western blot, their analysis and contributed to immunofluorescence experiments. MB, JM and MHJ contributed in qRT-PCR analysis and amelioration of the manuscript. MC, MB, RP and $C L$ contributed in analyzed, in vivo measurements and cell culture experiments. SP helps in manuscript criticism and collaborates for human tissue experiments. SB designed the study, supervised the overall study and wrote the manuscript. All authors have read and approved the manuscript.

\section{Competing interests}

The authors declare that they have no competing interests.

Received: 15 April 2011 Accepted: 27 September 2011 Published: 27 September 2011

\section{References}

1. Christman BW, McPherson CD, Newman JH, King GA, Bernard GR, Groves BM, Loyd JE: An imbalance between the excretion of thromboxane and prostacyclin metabolites in pulmonary hypertension. N Engl J Med 1992, 327(2):70-75.

2. Steudel W, Ichinose F, Huang PL, Hurford WE, Jones RC, Bevan JA, Fishman MC, Zapol WM: Pulmonary vasoconstriction and hypertension in mice with targeted disruption of the endothelial nitric oxide synthase (NOS 3) gene. Circ Res 1997, 81(1):34-41.

3. Stewart DJ, Levy RD, Cernacek P, Langleben D: Increased plasma endothelin-1 in pulmonary hypertension: marker or mediator of disease? Ann Intern Med 1991, 114(6):464-469.

4. Perros F, Montani D, Dorfmuller P, Durand-Gasselin I, Tcherakian C, Le Pavec J, Mazmanian M, Fadel E, Mussot S, Mercier O, et al: Platelet-derived growth factor expression and function in idiopathic pulmonary arterial hypertension. Am J Respir Crit Care Med 2008, 178(1):81-88.

5. Bonnet S, Michelakis ED, Porter CJ, Andrade-Navarro MA, Thebaud B, Bonnet S, Haromy A, Harry G, Moudgil R, McMurtry MS, et al: An abnormal mitochondrial-hypoxia inducible factor- 1 alpha-Kv channel pathway disrupts oxygen sensing and triggers pulmonary arterial hypertension in fawn hooded rats: similarities to human pulmonary arterial hypertension. Circulation 2006, 113(22):2630-2641.

6. Bonnet S, Rochefort G, Sutendra G, Archer SL, Haromy A, Webster L, Hashimoto K, Bonnet SN, Michelakis ED: The nuclear factor of activated T cells in pulmonary arterial hypertension can be therapeutically targeted. Proc Natl Acad Sci USA 2007, 104(27):11418-11423.

7. McMurtry MS, Archer SL, Altieri DC, Bonnet S, Haromy A, Harry G, Bonnet S, Puttagunta L, Michelakis ED: Gene therapy targeting survivin selectively induces pulmonary vascular apoptosis and reverses pulmonary arterial hypertension. J Clin Invest 2005, 115(6):1479-1491.

8. Yildiz P: Molecular mechanisms of pulmonary hypertension. Clin Chim Acta 2009, 403(1-2):9-16.

9. Dumas de la Roque E, Savineau JP, Bonnet S: Dehydroepiandrosterone: A new treatment for vascular remodeling diseases including pulmonary arterial hypertension. Pharmacol Ther 2010, 126(2):186-199.

10. Dupuis J, Hoeper MM: Endothelin receptor antagonists in pulmonary arterial hypertension. Eur Respir J 2008, 31(2):407-415.

11. Li B, Yang L, Shen J, Wang C, Jiang Z: The antiproliferative effect of sildenafil on pulmonary artery smooth muscle cells is mediated via upregulation of mitogen-activated protein kinase phosphatase-1 and degradation of extracellular signal-regulated kinase $1 / 2$ phosphorylation. Anesth Analg 2007, 105(4):1034-1041, table of contents.

12. Ghofrani HA, Seeger W, Grimminger F: Imatinib for the treatment of pulmonary arterial hypertension. N Engl J Med 2005, 353(13):1412-1413.

13. Archer S, Rich S: Primary pulmonary hypertension: a vascular biology and translational research "Work in progress". Circulation 2000, 102(22):2781-2791.

14. Dong JT, Chen C: Essential role of KLF5 transcription factor in cell proliferation and differentiation and its implications for human diseases. Cell Mol Life Sci 2009, 66(16):2691-2706.

15. Liu R, Zheng HQ, Zhou Z, Dong JT, Chen C: KLF5 promotes breast cell survival partially through fibroblast growth factor-binding protein 1pERK-mediated dual specificity MKP-1 protein phosphorylation and stabilization. J Biol Chem 2009, 284(25):16791-16798.

16. Chen $C$, Benjamin MS, Sun $X$, Otto KB, Guo P, Dong XY, Bao Y, Zhou $Z$, Cheng $X$, Simons JW, et al: KLF5 promotes cell proliferation and tumorigenesis through gene regulation and the TSU-Pr1 human bladder cancer cell line. Int J Cancer 2006, 118(6):1346-1355.

17. Sakamoto $H$, Sakamaki T, Kanda T, Hoshino $Y$, Sawada $Y$, Sato M, Sato H, Oyama $Y$, Nakano A, Takase $S$, et al: Smooth muscle cell outgrowth from 
coronary atherectomy specimens in vitro is associated with less time to restenosis and expression of a key Transcription factor KLF5/BTEB2. Cardiology 2003, 100(2):80-85.

18. Nagai R, Suzuki T, Aizawa K, Shindo T, Manabe I: Significance of the transcription factor KLF5 in cardiovascular remodeling. J Thromb Haemost 2005, 3(8):1569-1576.

19. Shindo T, Manabe I, Fukushima Y, Tobe K, Aizawa K, Miyamoto S, KawaiKowase K, Moriyama N, Imai Y, Kawakami H, et al: Kruppel-like zinc-finger transcription factor KLF5/BTEB2 is a target for angiotensin II signaling and an essential regulator of cardiovascular remodeling. Nat Med 2002, 8(8):856-863.

20. Bonnet S, Archer SL, Allalunis-Turner J, Haromy A, Beaulieu C, Thompson R, Lee $C T$, Lopaschuk GD, Puttagunta L, Bonnet $S$, et al: A mitochondria-K+ channel axis is suppressed in cancer and its normalization promotes apoptosis and inhibits cancer growth. Cancer Cell 2007, 11(1):37-51.

21. Bonnet $S$, Paulin R, Sutendra G, Dromparis P, Roy M, Watson KO, Nagendran J, Haromy A, Dyck JR, Michelakis ED: Dehydroepiandrosterone reverses systemic vascular remodeling through the inhibition of the Akt/ GSK3-\{beta\}/NFAT axis. Circulation 2009, 120(13):1231-1240.

22. Boengler K, Hilfiker-Kleiner D, Drexler H, Heusch G, Schulz R: The myocardial JAK/STAT pathway: from protection to failure. Pharmacol Ther 2008, 120(2):172-185

23. Grote K, Luchtefeld M, Schieffer B: JANUS under stress-role of JAK/STAT signaling pathway in vascular diseases. Vascul Pharmacol 2005, 43(5):357-363.

24. Darnell JE Jr: STATs and gene regulation. Science 1997, 277(5332):1630-1635

25. Banes-Berceli AK, Ketsawatsomkron P, Ogbi S, Patel B, Pollock DM, Marrero MB: Angiotensin II and endothelin-1 augment the vascular complications of diabetes via JAK2 activation. Am J Physiol Heart Circ Physiol 2007, 293(2):H1291-1299.

26. Csiszar A, Labinskyy N, Olson S, Pinto JT, Gupte S, Wu JM, Hu F, Ballabh P, Podlutsky A, Losonczy G, et al: Resveratrol prevents monocrotalineinduced pulmonary hypertension in rats. Hypertension 2009, 54(3):668-675.

27. Schermuly RT, Dony E, Ghofrani HA, Pullamsetti S, Savai R, Roth M, Sydykov A, Lai YJ, Weissmann N, Seeger W, et al: Reversal of experimental pulmonary hypertension by PDGF inhibition. J Clin Invest 2005, 115(10):2811-2821

28. Frasch HF, Marshall C, Marshall BE: Endothelin-1 is elevated in monocrotaline pulmonary hypertension. Am J Physiol 1999, 276(2 Pt 1): L304-310.

29. Bourillot PY, Aksoy I, Schreiber V, Wianny F, Schulz H, Hummel O, Hubner N Savatier P: Novel STAT3 target genes exert distinct roles in the inhibition of mesoderm and endoderm differentiation in cooperation with Nanog. Stem Cells 2009, 27(8):1760-1771.

30. Amirak E, Zakkar M, Evans PC, Kemp PR: Perfusion of veins at arterial pressure increases the expression of KLF5 and cell cycle genes in smooth muscle cells. Biochem Biophys Res Commun 2010, 391(1):818-823.

31. Nandan MO, Chanchevalap S, Dalton WB, Yang WW: Kruppel-like factor 5 promotes mitosis by activating the cyclin B1/Cdc2 complex during oncogenic Ras-mediated transformation. FEBS Lett 2005, 579(21):4757-4762.

32. Yang $Y$, Goldstein BG, Chao HH, Katz JP: KLF4 and KLF5 regulate proliferation, apoptosis and invasion in esophageal cancer cells. Cancer Biol Ther 2005, 4(11):1216-1221.

33. He M, Han M, Zheng B, Shu YN, Wen JK: Angiotensin II stimulates KLF5 phosphorylation and its interaction with c-Jun leading to suppression of p21 expression in vascular smooth muscle cells. J Biochem 2009, 146(5):683-691.

34. Fujiu K, Manabe I, Ishihara A, Oishi Y, Iwata H, Nishimura G, Shindo T, Maemura K, Kagechika H, Shudo K, et al: Synthetic retinoid Am80 suppresses smooth muscle phenotypic modulation and in-stent neointima formation by inhibiting KLF5. Circ Res 2005, 97(11):1132-1141.

35. McMurtry MS, Archer SL, Altieri DC, Bonnet S, Haromy A, Harry G, Puttagunta L, Michelakis ED: Gene therapy targeting survivin selectively induces pulmonary vascular apoptosis and reverses pulmonary arterial hypertension. J Clin Invest 2005, 115(6):1479-1491.

36. Montorsi M, Maggioni M, Falleni M, Pellegrini C, Donadon M, Torzilli G, Santambrogio R, Spinelli A, Coggi G, Bosari S: Survivin gene expression in chronic liver disease and hepatocellular carcinoma. Hepatogastroenterology 2007, 54(79):2040-2044.
37. Murakami M, Sakai H, Kodama A, Mori T, Maruo K, Yanai T, Masegi T: Expression of the anti-apoptotic factors $\mathrm{Bcl}-2$ and survivin in canine vascular tumours. J Comp Pathol 2008, 139(1):1-7.

38. Ryan BM, Konecny GE, Kahlert S, Wang HJ, Untch M, Meng G, Pegram MD, Podratz KC, Crown J, Slamon DJ, et al: Survivin expression in breast cancer predicts clinical outcome and is associated with HER2, VEGF, urokinase plasminogen activator and PAI-1. Ann Oncol 2006, 17(4):597-604.

39. Sakao S, Taraseviciene-Stewart L, Lee JD, Wood K, Cool CD, Voelkel NF: Initial apoptosis is followed by increased proliferation of apoptosisresistant endothelial cells. Faseb J 2005.

40. Zhu N, Gu L, Findley HW, Chen C, Dong JT, Yang L, Zhou M: KLF5 Interacts with p53 in regulating survivin expression in acute lymphoblastic leukemia. J Biol Chem 2006, 281(21):14711-14718.

41. Wan H, Luo F, Wert SE, Zhang L, Xu Y, Ikegami M, Maeda Y, Bell SM, Whitsett JA: Kruppel-like factor 5 is required for perinatal lung morphogenesis and function. Development 2008, 135(15):2563-2572.

42. Toshner M, Voswinckel R, Southwood M, Al-Lamki R, Howard LS, Marchesan D, Yang J, Suntharalingam J, Soon E, Exley A, et al: Evidence of dysfunction of endothelial progenitors in pulmonary arterial hypertension. Am J Respir Crit Care Med 2009, 180(8):780-787.

43. Kumekawa M, Fukuda G, Shimizu S, Konno K, Odawara M: Inhibition of monocyte chemoattractant protein- 1 by Kruppel-like factor 5 small interfering RNA in the tumor necrosis factor- alpha-activated human umbilical vein endothelial cells. Biol Pharm Bull 2008, 31(8):1609-1613.

44. Zhan Y, Brown C, Maynard E, Anshelevich A, Ni W, Ho IC, Oettgen P: Ets-1 is a critical regulator of Ang II-mediated vascular inflammation and remodeling. J Clin Invest 2005, 115(9):2508-2516.

45. Aizawa K, Suzuki T, Kada N, Ishihara A, Kawai-Kowase K, Matsumura T, Sasaki K, Munemasa Y, Manabe I, Kurabayashi M, et al: Regulation of platelet-derived growth factor-A chain by Kruppel-like factor 5: new pathway of cooperative activation with nuclear factor-kappaB. J Biol Chem 2004, 279(1):70-76.

46. Rehman J, Archer SL: A proposed mitochondrial-metabolic mechanism for initiation and maintenance of pulmonary arterial hypertension in fawn-hooded rats: the Warburg model of pulmonary arterial hypertension. Adv Exp Med Biol 2010, 661:171-185.

47. Mori A, Moser C, Lang SA, Hackl C, Gottfried E, Kreutz M, Schlitt HJ, Geissler EK, Stoeltzing O: Up-regulation of Kruppel-like factor 5 in pancreatic cancer is promoted by interleukin-1 beta signaling and hypoxia-inducible factor-1 alpha. Mol Cancer Res 2009, 7(8):1390-1398.

48. Cheng Y, Liu X, Yang J, Lin Y, Xu DZ, Lu Q, Deitch EA, Huo Y, Delphin ES, Zhang C: MicroRNA-145, a novel smooth muscle cell phenotypic marker and modulator, controls vascular neointimal lesion formation. Circ Res 2009, 105(2):158-166.

49. Caruso P, Maclean MR, Khanin R, McClure J, Soon E, Southwood M, McDonald RA, Greig JA, Robertson KE, Masson R, et al: Dynamic Changes in Lung MicroRNA Profiles During the Development of Pulmonary Hypertension Due to Chronic Hypoxia and Monocrotaline. Arterioscler Thromb Vasc Biol 2010

50. Courboulin A, Paulin R, Giguere NJ, Saksouk N, Perreault T, Meloche J, Paquet ER, Biardel S, Provencher S, Cote J, et al: Role for miR-204 in human pulmonary arterial hypertension. J Exp Med 2011.

51. Paulin R, Courboulin A, Meloche J, Mainguy V, Dumas de la Roque E, Saksouk N, Cote J, Provencher S, Sussman MA, Bonnet S: Signal transducers and activators of transcription-3/pim1 axis plays a critical role in the pathogenesis of human pulmonary arterial hypertension. Circulation 2011, 123(11):1205-1215.

52. Sehgal PB, Mukhopadhyay S, Patel K, Xu F, Almodovar S, Tuder RM, Flores SC: Golgi dysfunction is a common feature in idiopathic human pulmonary hypertension and vascular lesions in SHIV-nef-infected macaques. Am J Physiol Lung Cell Mol Physiol 2009, 297(4):L729-737.

53. Brock M, Trenkmann M, Gay RE, Michel BA, Gay S, Fischler M, Ulrich S, Speich R, Huber LC: Interleukin- 6 modulates the expression of the bone morphogenic protein receptor type II through a novel STAT3-microRNA cluster 17/92 pathway. Circ Res 2009, 104(10):1184-1191.

doi:10.1186/1465-9921-12-128

Cite this article as: Courboulin et al:: Krüppel-like Factor 5 contributes to pulmonary artery smooth muscle proliferation and resistance to apoptosis in human pulmonary arterial hypertension. Respiratory Research 2011 12:128. 